\title{
Clinical Research Skills Assessment: An Investigation into the Determinants of Effective Research
}

Mohamad Al-Tannir ${ }^{1}$, Isamme AlFayyad ${ }^{1}$, Amani Abu-Shaheen ${ }^{1}$, Ahmed Al-Badr ${ }^{1}$, Fatimah Al Mousawi ${ }^{1}$

1. Research Center, King Fahad Medical City

$\square$ Corresponding author: Mohamad Al-Tannir, maltannir@kfmc.med.sa

Disclosures can be found in Additional Information at the end of the article

\section{Abstract}

\section{Background}

Developing individual research skills and enhancing the institutional research culture leads to quality research capabilities and research excellence at the national level. We aim to assess the educational needs of healthcare providers regarding research skills at King Fahad Medical City (KFMC), Riyadh, Saudi Arabia.

\section{Methods}

From February 2016 to October 2016, we conducted a cross-sectional study using a selfadministered questionnaire of the healthcare providers at KFMC. The questionnaire targeted staff who have not been involved in research ( $n=353$; “category-1"), staff who received Institutional Review Board (IRB) approval (n=94; “category-2”), and staff who have completed and published their research ( $\mathrm{n}=53$; “category-3"). A descriptive analysis was used to measure the frequency, and the chi-square test was used to test significance when comparing categorical data.

\section{Results}

The final analysis contained 500 questionnaires. The number of women was higher than that of men in "category-2 "and "category-3" (53.2\%, 62.3\%), respectively. Approximately $62.4 \%$ of “category-1" participants reported good, poor, and very poor knowledge of epidemiology. Participants in "category-1" and "category-2" stated poor and very poor levels when writing a manuscript (43\%, 23.4\%), respectively. Only $37 \%$ of participants in "category-3" showed very good to excellent research skills. However, there was a significant correlation between the mean scores of research skill and research stage ( $p$-value $<0.001)$.

Received 10/01/2017 Review began 11/06/2017 Review ended 11/20/2017 Published 11/22/2017

C Copyright 2017

Al-Tannir et al. This is an open access article distributed under the terms of the Creative Commons Attribution License CC-BY 3.0., which permits unrestricted use, distribution, and reproduction in any medium, provided the original author and source are credited.

\section{Conclusion}

The results showed a significant variation in research skills needs among research stage categories; therefore, meeting the educational needs of healthcare providers aimed at effective research shall be constructed based on their stage of research.

Categories: Epidemiology/Public Health

Keywords: educational needs, health care providers, knowledge, research, saudi arabia 


\section{Introduction}

Recently, there was an increasing focus to boost health research and evidence-based practice to achieve the national health goals, primarily in developing countries. A robust health research system is significant for an efficient health system [1]. Moreover, clinical research influences the prevention, diagnosis, and treatment of diseases [2].

The development of research capacity is primarily based on the development of individual research skills and the enhancement of institutional research culture, which ultimately leads to quality research capacity and research excellence at the national level [3]. An appraisal of the research skills of developing researchers has shown that some significant research skills are essential for competence in conducting research. These research skills include, but are not limited to, information seeking, communicating (e.g., protocols submission and manuscript writing skills), methodological skills, and data analysis (e.g., correct data analysis and statistics) [4].

Vigorous and energetic research personnel are imperative to Saudi's future prosperity in the research arena. Our researchers and research scientists constitute crucial talent that needs to be acknowledged and supported. King Fahad Medical City (KFMC), as a reference medical care institution, provides specialized patient care by being active in training, education, and research. These activities are mutually connected and inter-reliant to build an effective research environment, support current research investigators, and establish a platform for future research scholars. In addition, continuous professional academic achievements are associated with research skills training and experience in the early periods of an individual's professional life [5-6].

Numerous studies have been conducted to assess the research skills and knowledge of nurses in the healthcare system. A previous study surveyed more than 400 nurses in six different health care settings within the UK and showed that approximately $93 \%$ of the nurses were not satisfied with their research knowledge and skills [7]. Moreover, Pravikoff et al. and Koehn et al. revealed a deficiency of skills among nurses to search, appraise, and produce research literature, a difficulty in understanding research papers, and an absence of knowledge/partial knowledge of research [8-9].

Research experience is vital to a healthcare provider's evidence-based practice, as it conveys skills such as literature review and data collection and analysis, and evidence of critical appraisal [10-11]. In a national mailed study in Canada, Leahy et al. found that family physicians varied in their ideas regarding the significance of research education during residency [12].

The literature recommends that professional learning and development is continued when the education is allied with the practice, whereby individual motivation energizes the educational work [13]. Therefore, we propose that the first step in promoting the research culture and practice is to assess the educational needs of health care providers and plan a continuous process of learning.

Few studies have examined the importance of evolving a researcher's skills. Kardash designed a tool to capture the evolving research skills of undergraduates involved in research activities [14]. In addition, Powers and Enright created an instrument to measure graduate student research skills [15].

Given the need to develop an operational plan and a distinctive, effective research program that recognizes the vital role research personnel play in supporting quality discoveries and 
advances, a survey was conducted to assess the educational needs of healthcare providers regarding research knowledge and skills at KFMC.

\section{Materials And Methods}

\section{Study design}

Following Institutional Review Board (IRB) approval, a cross-sectional study was conducted at KFMC using a self-administered questionnaire from February 2016 to October 2016.

\section{Study participants and sample size}

The questionnaire targeted three categories among healthcare providers, according to their stage of research activity, including 1) staff who have never been involved in research activities "category-1", 2) staff who have received IRB approval but have not finished their projects "category-2", and 3) staff who have completed and published their research "category-3." The sample size was calculated according to research categories. The sample size of "category-1" was based on the total number of healthcare providers at KFMC (353 participants). The "category-2" sample size was calculated based on the number of approved studies from the IRB office, which was found to be 94 participants. The sample size of "category-3" was calculated based on the number of completed and published studies through the KFMC research center (53 participants). Participants were approached by a trained research assistant and asked to sign informed consent forms and to complete a self-administered questionnaire. Verbal consent was obtained from each participant before filling out the survey.

\section{Data collection methods, instruments, and measurements}

The questionnaire was developed after an in-depth review of the literature. Before commencing work, a pilot study was conducted to assess the reliability of the questionnaire. Cronbach's alpha resulted in more than 0.9 .

The questionnaire was constructed in three parts. Part one was composed of participant characteristics, including age, gender, department (Women Specialized Hospital (WSH); Children Hospital (CH); Rehabilitation Hospital (RH); Main Hospital, Comprehensive Cancer Center (CCC); King Salman Heart Center (KSHC); National Neuroscience Institute (NNI); Obesity, Metabolic and Endocrine Center (OMEC); Out Patient Department (OPD), and other ancillary services), profession, and occupation. Part two looked at the level and origin of the academic degree, total years of experience, and involvement at KFMC. The third part addressed the stages of research activity, the number of research participants, and the assessment of skills needed for effective research. The assessment of the participant's skills was assessed using a five-point Likert scale, ranging from "Excellent - 1", "Very Good - 2", “Good - 3", "Poor - 4", and "Very Poor - 5." The research skills questions evaluated the following areas: 1) basic concepts of research and epidemiology, 2) appraisal of medical literature, 3) effective literature search, 4) basics of biostatistics, 5) writing a grant proposal, 6) writing a scientific manuscript, 7) submitting a manuscript to a journal, 8) addressing journal reviewers comments, 9) research monitoring, 10) presentation skills (e.g., oral vs. poster), 11) communication skills, 12) data records and management, and 13) knowledge of good clinical practice guidelines (GCP). A mean research skills score of $\leqslant 2$ (very good and excellent) was considered a satisfactory research skill level; higher scores were considered unsatisfactory.

\section{Statistical analysis}

Data analysis was conducted using SPSS 22.0 software (SPSS Inc., Chicago, IL, USA). The arithmetic mean was used as a summary to assess the level of research skills. The chi-square test was used to determine the strength of the association between the categories of research 


\section{Cureus}

stages and research skills. For all statistical analysis, $p \leqslant 0.05$ was considered statistically

significant.

\section{Results}

A total of 500 questionnaires were collected and entered into the final data analysis. Table 1 shows the participant's demographics and characteristics. A total of 353 (70.6\%) participants have not been involved in any research activity: "category-1.” The majority of participants in “category-2" and "category-3" (90.1\% and 81.1\%, respectively) have participated in fewer than five studies. Among all categories, more than two-thirds of the participants were younger than 40 years of age. Women were more active in research than men in "category-2" and "category$3 "$ (53.2\% and 62.3\%, respectively).

\begin{tabular}{|c|c|c|c|c|c|}
\hline & & \multicolumn{4}{|l|}{ Stage of research } \\
\hline & & $\begin{array}{l}\text { Never involved in } \\
\text { research n (\%) }\end{array}$ & approval n (\%) & $\begin{array}{l}\text { Completed and } \\
\text { published research n } \\
(\%)\end{array}$ & $\begin{array}{l}\mathrm{p} \text { - } \\
\text { values }\end{array}$ \\
\hline \multicolumn{2}{|l|}{ Stage of research activity } & $353(70.6)$ & $94(18.8)$ & 53 (10.6) & \\
\hline \multirow{4}{*}{$\begin{array}{l}\text { How many research studies } \\
\text { have you participated in? }\end{array}$} & $1-5$ & 0 & $73(90.1)$ & $43(81.1)$ & \\
\hline & $5-10$ & 0 & $5(6.2)$ & $2(3.8)$ & \\
\hline & $>10$ & 0 & $3(3.7)$ & $8(15.1)$ & \\
\hline & Total & $353(100)$ & $81(100)$ & $53(100)$ & \\
\hline \multirow{5}{*}{ Age } & $\leq 30$ & $144(45.9)$ & $27(30.7)$ & $18(36.7)$ & \multirow{5}{*}{0.065} \\
\hline & $31-40$ & $100(31.8)$ & $43(48.9)$ & $22(44.9)$ & \\
\hline & $41-50$ & $62(19.7)$ & $15(17.0)$ & $7(14.3)$ & \\
\hline & $>50$ & $8(2.5)$ & $3(3.4)$ & $2(4.1)$ & \\
\hline & Total & $314(100)$ & $88(100)$ & $49(100)$ & \\
\hline \multirow{3}{*}{ Gender } & Male & $48(13.8)$ & $44(46.8)$ & $20(37.7)$ & \multirow{3}{*}{$<0.001$} \\
\hline & Female & 301 (86.2) & $50(53.2)$ & $33(62.3)$ & \\
\hline & Total & $349(100)$ & $94(100)$ & $53(100)$ & \\
\hline \multirow{6}{*}{ Department } & WSH & $69(20.1)$ & $7(7.9)$ & $10(23.3)$ & \\
\hline & $\mathrm{CH}$ & $16(4.7)$ & $1(1.1)$ & $3(7.0)$ & \\
\hline & RH & $61(17.7)$ & $6(6.7)$ & $5(11.6)$ & \\
\hline & $\begin{array}{l}\text { Main } \\
\text { hospital }\end{array}$ & 68 (19.8) & $23(25.8)$ & $6(14.0)$ & \\
\hline & $\mathrm{CCC}$ & $31(9.0)$ & $10(11.2)$ & $4(9.3)$ & \multirow{2}{*}{0.002} \\
\hline & KSHC & $18(5.2)$ & 14 (15.7) & $3(7.0)$ & \\
\hline
\end{tabular}




\section{Cureus}

B.Sc. degree origin

$\begin{array}{llll}\text { NNI } & 17(4.9) & 11(12.4) & 6(14.0) \\ \text { OMEC } & 17(4.9) & 4(4.5) & 2(4.7) \\ \text { OPD } & 0(0.0) & 0(0.0) & 0(0.0)\end{array}$

M.Sc. degree origin

$$
\text { Ot }
$$

Others $\quad 47(13.7)$

13 (14.6)

Total $\quad 344(100)$

$89(100)$

$43(100)$

Middle

81 (27.6)

$53(61.6)$

19 (38.8)

East

South/Far

East

212 (72.1)

24 (27.9)

23 (46.9)

$<0.001$

West $\quad 1(0.3)$

$9(10.5)$

$7(14.3)$

Total $294(100)$

$86(100)$

49 (100)

Middle

East

$26(70.3)$

$19(47.5)$

$7(30.4)$

South/Far

East

$9(24.3)$

$5(12.5)$

$6(26.1)$

0.002

Ph.D. degree origin

West

$2(5.4)$

$16(40.0)$

10 (43.5)

Total $37(100)$

$40(100)$

$23(100)$

Middle

East

$5(41.7)$

$0(0.0)$

South/Far

7 (58.3)

2 (16.7)

$1(20.0)$

0.009

Profession

$$
\text { East }
$$

$0(0.0)$

$5(41.7)$

$4(80.0)$

Total

12(100)

$12(100)$

$5(100)$

Physician 45 (13.0)

38 (43.2)

15 (33.3)

Nurse $\quad 284(81.8)$

34 (38.6)

24 (53.3)

Pharmacist $14(4.0)$

12 (13.6)

$3(6.7)$

$<0.001$

Laboratory

Specialist

4 (1.2)

$4(4.5)$

$3(6.7)$

Total

$347(100)$

$88(100)$

$45(100)$

Consultant $2(1.0)$

14 (20.6)

$8(20.5)$

Assistant

$9(4.5)$

12 (17.6)

$3(7.7)$

Fellow $\quad 17(8.4)$

$5(7.4)$

$4(10.3)$

Resident 25 (12.4)

$6(8.8)$

1 (2.6)

Occupation 


\section{Cureus}

\begin{tabular}{|c|c|c|c|c|}
\hline & Head nurse & $143(70.8)$ & $22(32.4)$ & $17(43.6)$ \\
\hline & $\begin{array}{l}\text { Senior } \\
\text { Pharmacist }\end{array}$ & $3(1.5)$ & $7(10.3)$ & $3(7.7)$ \\
\hline & $\begin{array}{l}\text { Senior lab } \\
\text { Specialist }\end{array}$ & $3(1.5)$ & $2(2.9)$ & $3(7.7)$ \\
\hline & Total & 202 (100) & $68(100)$ & $39(100)$ \\
\hline \multirow{4}{*}{ Total years of experience } & $<5$ & $92(26.4)$ & $24(25.5)$ & $15(28.8)$ \\
\hline & $5-10$ & $129(37.1)$ & $29(30.9)$ & $18(34.6)$ \\
\hline & $\geq 10$ & $127(36.5)$ & $41(43.6)$ & $19(36.5)$ \\
\hline & Total & 348 & 94 & 52 \\
\hline \multirow{4}{*}{$\begin{array}{l}\text { Years of experience at } \\
\text { KFMC }\end{array}$} & $<5$ & $199(58.9)$ & $42(45.2)$ & $37(71.2)$ \\
\hline & $5-10$ & $90(26.6)$ & $35(37.6)$ & $11(21.2)$ \\
\hline & $\geq 10$ & $49(14.5)$ & $16(17.2)$ & $4(7.7)$ \\
\hline & Total & 338 (100) & $9(100)$ & $52(100)$ \\
\hline
\end{tabular}

Abbreviations: CCC, Comprehensive Cancer Center; CH, Children Hospital; IRB, Institutional Review Board; KFMC, King Fahad Medical City; KSHC, King Salman Heart Center; NNI, National Neuroscience Institute; OMEC, Obesity, Metabolic and Endocrine Center; OPD, Outpatient Department; RH, Rehabilitation Hospital; WSH, Women Specialized Hospital.

\section{TABLE 1: Characteristics of participants}

Our results revealed that among bachelor degree holders, more staff from the Middle East (61.6\%) were involved in "category-2" and more staff from the South/Far East (46.9\%) were involved in "category-3." Among staff with Ph.D. degrees, Middle Easterners and Westerners displayed similar involvement in "category-2"; however, staff with Western certificates were more involved in "category-3." Participating physicians were mainly grouped in "category-2" and "category-3"; meanwhile, the majority of nurses (81.8\%) have never been involved in a research activity. Staff with more than 10 years total experience were grouped in "category-2" and "category-3" (43.6\% and 36.5\%, respectively); however, staff with under five years of experience at KFMC were placed in "category-2" (45.2\%) and "category-3" (71.1\%). A statistically significant association was found between participant gender, department, origin of academic degree, profession, occupation, and years of experience at KFMC (Table 1).

Table 2 shows participants' research skill levels. Approximately $62.4 \%$ of "category-1" participants reported good, poor, or very poor (combined) knowledge of the basic concepts of epidemiology. However, more than two-thirds of participants in "category-2" and "category-3" reported excellent or very good (combined) levels of research skills. A total of $49.5 \%$ of participants in "category-1" reported that they have an excellent or very good (combined) level of medical literature review. However, participants in "category-2" and "category-3" have a better understanding of the medical literature ( $76.6 \%$ and $67.9 \%$, respectively) compared with "category-1." "Category-1" participants were average in effective literature searches (50.6\% scored good, poor, or very poor). Furthermore, 30.2\% of participants in "category-2" and 32.1\% 


\section{Cureus}

in "category-3" reported good, poor, or very poor (combined) research skills in effective literature searching. Our results determined that statistical analysis skills were higher in "category-2" and "category-3" participants.

\begin{tabular}{|c|c|c|c|c|}
\hline & & \multicolumn{3}{|l|}{ Stage of research } \\
\hline & & Category-1 n(\%) & Category-2 n(\%) & Category-3 n(\%) \\
\hline \multirow{6}{*}{ Basic concepts of research and epidemiology } & Excellent & $19(5.4)$ & $25(26.6)$ & $11(20.8)$ \\
\hline & Very good & 113 (32.2) & 37 (39.4) & $23(43.4)$ \\
\hline & Good & $212(60.4)$ & $31(33.0)$ & $19(35.8)$ \\
\hline & Poor & $6(1.7)$ & $1(1.1)$ & $0(0.0)$ \\
\hline & Very poor & $1(0.3)$ & $0(0.0)$ & $0(0.0)$ \\
\hline & Total & $351(100)$ & $94(100)$ & $53(100)$ \\
\hline \multirow{6}{*}{ Appraisal of medical literature } & Excellent & $24(6.9)$ & $28(29.8)$ & $12(22.6)$ \\
\hline & Very good & 149 (42.6) & $44(46.8)$ & $24(45.3)$ \\
\hline & Good & $171(48.9)$ & $19(20.2)$ & $17(32.1)$ \\
\hline & Poor & $5(1.4)$ & $3(3.2)$ & $0(0.0)$ \\
\hline & Very poor & $1(0.3)$ & $0(0.0)$ & $0(0.0)$ \\
\hline & Total & $350(100)$ & $94(100)$ & $53(100)$ \\
\hline \multirow{6}{*}{ Effective literature search } & Excellent & 41 (11.6) & 30 (32.3) & $11(20.8)$ \\
\hline & Very good & $133(37.8)$ & $34(36.6)$ & 25 (47.2) \\
\hline & Good & $166(47.2)$ & $28(30.1)$ & 16 (30.2) \\
\hline & Poor & $11(3.1)$ & $1(1.1)$ & $1(1.9)$ \\
\hline & Very poor & $1(0.3)$ & $0(0.0)$ & $0(0.0)$ \\
\hline & Total & 352 (100) & $93(100)$ & 53 (100) \\
\hline \multirow{8}{*}{ Basics of biostatistics } & Excellent & $17(5.0)$ & $8(9.2)$ & $8(15.1)$ \\
\hline & Very good & $103(30.3)$ & $45(51.7)$ & $21(39.6)$ \\
\hline & Good & 198 (58.2) & $31(35.6)$ & $22(41.5)$ \\
\hline & Poor & $21(6.2)$ & $3(3.4)$ & $2(3.8)$ \\
\hline & Very poor & $1(0.3)$ & $0(0.0)$ & $0(0.0)$ \\
\hline & Total & 340 (100) & $87(100)$ & $53(100)$ \\
\hline & Excellent & $2(0.6)$ & $2(2.1)$ & $9(17.0)$ \\
\hline & Very good & $28(8.0)$ & $26(27.7)$ & $14(26.4)$ \\
\hline
\end{tabular}




\section{Cureus}

Writing a grant proposal

Writing a scientific manuscript

Submitting a manuscript to a journal

Addressing journals' reviewer comments

Research monitoring

\begin{tabular}{|c|c|c|c|}
\hline Good & $165(47.3)$ & 46 (48.9) & $21(39.6)$ \\
\hline Poor & $145(41.5)$ & 17 (18.1) & 8 (15.1) \\
\hline Very poor & $9(2.6)$ & $3(3.2)$ & $1(1.9)$ \\
\hline Total & $349(100)$ & 94 (100) & $53(100)$ \\
\hline Excellent & $1(0.3)$ & $3(3.2)$ & $5(9.4)$ \\
\hline Very good & $26(7.4)$ & 29 (30.9) & 17 (32.1) \\
\hline Good & $155(44.4)$ & $40(42.6)$ & 24 (45.3) \\
\hline Poor & $157(45.0)$ & 18 (19.1) & 6 (11.3) \\
\hline Very poor & $10(2.9)$ & $4(4.3)$ & $1(1.9)$ \\
\hline Total & $349(100)$ & 94 (100) & $53(100)$ \\
\hline Excellent & $2(0.6)$ & $3(3.2)$ & $9(17.0)$ \\
\hline Very good & $20(5.8)$ & 18 (19.4) & 10 (18.9) \\
\hline Good & $146(42.2)$ & $34(36.6)$ & $24(45.3)$ \\
\hline Poor & $165(47.7)$ & 32 (34.4) & 8 (15.1) \\
\hline Very poor & $13(3.8)$ & $6(6.5)$ & $2(3.8)$ \\
\hline Total & $346(100)$ & $93(100)$ & $53(100)$ \\
\hline Excellent & $5(1.4)$ & $4(4.5)$ & $9(17.0)$ \\
\hline Very good & 25 (7.2) & 20 (22.5) & $11(20.8)$ \\
\hline Good & $172(49.6)$ & $41(46.1)$ & 24 (45.3) \\
\hline Poor & $136(39.2)$ & $23(25.8)$ & 8 (15.1) \\
\hline Very poor & $9(2.6)$ & $1(1.1)$ & $1(1.9)$ \\
\hline Total & 347 (100) & 89 (100) & $53(100)$ \\
\hline Excellent & $4(1.1)$ & $6(6.6)$ & $11(20.8)$ \\
\hline Very good & 44 (12.6) & 29 (31.9) & $18(34.0)$ \\
\hline Good & $187(53.7)$ & 43 (47.3) & $18(34.0)$ \\
\hline Poor & $111(31.9)$ & 12 (13.2) & 6 (11.3) \\
\hline Very poor & $2(0.6)$ & $1(1.1)$ & $0(0.0)$ \\
\hline Total & $348(100)$ & 91 (100) & $53(100)$ \\
\hline Excellent & $18(5.1)$ & 21 (22.3) & $14(26.4)$ \\
\hline Very good & 77 (21.9) & 41 (43.6) & 20 (37.7) \\
\hline Good & $212(60.4)$ & $26(27.7)$ & 17 (32.1) \\
\hline
\end{tabular}




\section{Cureus}

Presentation skills (oral vs. posters)

Communication skills

Data records and management

Knowledge of good clinical practice guidelines

\begin{tabular}{|c|c|c|c|}
\hline Poor & 41 (11.7) & $6(6.4)$ & 1 (1.9) \\
\hline Very poor & $3(0.9)$ & $0(0.0)$ & $1(1.9)$ \\
\hline Total & $351(100)$ & $94(100)$ & $53(100)$ \\
\hline Excellent & $31(8.8)$ & $33(35.1)$ & 15 (28.3) \\
\hline Very good & $123(34.9)$ & $41(43.6)$ & 24 (45.3) \\
\hline Good & $187(53.1)$ & $18(19.1)$ & $14(26.4)$ \\
\hline Poor & $9(2.6)$ & $2(2.1)$ & $0(0.0)$ \\
\hline Very poor & $2(0.6)$ & $0(0.0)$ & $0(0.0)$ \\
\hline Total & $352(100)$ & $94(100)$ & $53(100)$ \\
\hline Excellent & $18(5.1)$ & $26(28.6)$ & $13(24.5)$ \\
\hline Very good & $90(25.6)$ & 35 (38.5) & 21 (39.6) \\
\hline Good & $207(59.0)$ & $24(26.4)$ & $16(30.2)$ \\
\hline Poor & $33(9.4)$ & $6(6.6)$ & $3(5.7)$ \\
\hline Very poor & $3(0.9)$ & $0(0.0)$ & $0(0.0)$ \\
\hline Total & $351(100)$ & 91 (100) & $53(100)$ \\
\hline Excellent & 5 (1.5) & $8(8.9)$ & $12(24.0)$ \\
\hline Very good & 44 (13.0) & 39 (43.3) & $12(24.0)$ \\
\hline Good & $206(60.8)$ & $33(36.7)$ & $20(40.0)$ \\
\hline Poor & $74(21.8)$ & $9(10.0)$ & $4(8.0)$ \\
\hline Very poor & $10(2.9)$ & $1(1.1)$ & $2(4.0)$ \\
\hline Total & 339 (100) & 90 (100) & 50 (100) \\
\hline
\end{tabular}

\section{TABLE 2: Distribution of participants' responses for their level of research skills}

More than half (combined) of the participants among each of the three categories reported good or below in grant proposal writing skills. When participants were asked about their skill of writing a scientific manuscript, participants in "category-1" and "category-2" reported poor or very poor (43\% and $23.4 \%$, respectively). Nevertheless, only $13.2 \%$ of participants in "category3 " revealed poor or very poor levels in scientific manuscript writing. When asked about their skill at addressing a journal reviewer's comments, $17 \%$ of the participants in "category-3" indicated poor or very poor skills, and only $37 \%$ of participants in "category-3" showed very good or excellent skills.

Approximately $11.1 \%$ of participants in "category-1", $12 \%$ in "category-2", and $24.1 \%$ in "category-3" reported a poor or very poor understanding of GCP guidelines. In addition, Table 2 shows that there was a significant difference in research skill among the categories of 


\section{Cureus}

participants.

Table 3 shows that participants in "category-1" have an unsatisfactory level of all research skills (mean scale score $\geqslant 2$ ). Moreover, participants in "category-2" demonstrate satisfactory research skills in their appraisal of medical literature, effective literature searching, and communication skills. However, participants in "category-3," who have completed and published studies, display a satisfactory level of communication skills.

\begin{tabular}{|c|c|c|c|c|}
\hline & Category-1 & Category-2 & Category-3 & p-value \\
\hline & \multicolumn{3}{|l|}{ (Mean \pm SD) } & \\
\hline Basic concepts of epidemiology & $2.59 \pm 0.634$ & $2.09 \pm 0.799$ & $2.15 \pm 0.744$ & $<0.001$ \\
\hline Appraisal of medical literature & $2.46 \pm 0.657$ & $1.97 \pm 0.796^{\star}$ & $2.09 \pm 0.741$ & $<0.001$ \\
\hline Effective literature search & $2.43 \pm 0.748$ & $2.00 \pm 0.779^{\star}$ & $2.13 \pm 0.820$ & $<0.001$ \\
\hline Basics of biostatistics & $2.66 \pm 0.682$ & $2.33 \pm 0.693$ & $2.34 \pm 0.783$ & $<0.001$ \\
\hline Writing a grant proposal & $3.38 \pm 0.694$ & $2.93 \pm 0.820$ & $2.58 \pm 1.008$ & $<0.001$ \\
\hline Writing a scientific manuscript & $3.43 \pm 0.685$ & $2.90 \pm 0.893$ & $2.64 \pm 0.879$ & $<0.001$ \\
\hline Submitting a manuscript to a journal & $3.48 \pm 0.690$ & $3.22 \pm 0.942$ & $2.70 \pm 1.049$ & $<0.001$ \\
\hline Addressing journals' reviewer comments & $3.34 \pm 0.714$ & $2.97 \pm 0.845$ & $2.64 \pm 1.002$ & $<0.001$ \\
\hline Research monitoring & $3.18 \pm 0.695$ & $2.70 \pm 0.823$ & $2.36 \pm 0.942$ & $<0.001$ \\
\hline Presentation skills (oral vs. posters) & $2.81 \pm 0.736$ & $2.18 \pm 0.855$ & $2.15 \pm 0.907$ & $<0.001$ \\
\hline Communication skills & $2.51 \pm 0.716$ & $1.88 \pm 0.788^{*}$ & $1.98 \pm 0.747^{\star}$ & $<0.001$ \\
\hline Data records and management & $2.75 \pm 0.728$ & $2.11 \pm 0.900$ & $2.17 \pm 0.871$ & $<0.001$ \\
\hline Knowledge of GCP & $3.12 \pm 0716$ & $2.51 \pm 0.838$ & $2.44 \pm 1.072$ & $<0.001$ \\
\hline
\end{tabular}

\section{TABLE 3: Correlation of mean research skill scores with research stage categories}

*Satisfactory research skills mean score ( $\leq 2$ out of 5 )

Abbreviation: GCP, good clinical practices.

\section{Discussion}

In this cross-sectional study, we aimed to assess the educational needs of healthcare providers regarding research skills. Our results showed that there is a significant variation in research knowledge and skills among participants based on experience in the three categories of research stages.

The pattern of research productivity varies among researchers of different age and gender [1618]. The results of our study at KFMC revealed that participants in "category-2" and "category- 
3 " were under age 40, and that research productivity decreased with age, though the decline was statistically insignificant $(\mathrm{p}=0.065)$. Moreover, we found that women were more involved in research activities than men. However, a study conducted to assess the impact of gender on research productivity showed that the academic women's research is equal to men's productivity [19]. Therefore, we assume that age and gender are influencing factors in research productivity.

We noticed a negative trend between years of experience at KFMC and research activity among participants in “category-2" and “category-3," where research activity declined as years of experience increased.

Research requires quality skills in scientific manuscript writing that leads to publication in an indexed journal. The most important research skill required is an understanding of the basic concepts of epidemiology. Adequate knowledge of epidemiological methods is essential for conducting a study and analyzing data derived from clinical studies [20]. The results of the present study revealed that approximately one-third of the participants in "category-2" and "category-3" do not have a high quality (i.e., very good and excellent) level of the basic concepts of epidemiology, which certainly will undermine the quality of the research produced. Healthcare providers are challenged with an overabundance of scientific papers that focus on various clinical questions and subjects, evaluating clinical remedies, and discovering the predictive value of several factors in therapeutic outcomes [20]. This practice requires a considerable level of skill by the reader to research and appraise the study design, research methodology, data analysis, and interpretation of findings of pertinent research to reach conclusions. We examined the skills of medical literature search and appraisal and found that it was at an unsatisfactory level in 50\% of the participants in "category-1." Meanwhile, about twothirds of the participants in "category-2" and "category-3" have a higher research skill level in medical literature search and appraisal, which could be explained by their exposure to research and their research experience.

In healthcare disciplines, understanding statistical analysis has essential effects in modifying clinical practice, as it has a great implication on evidence-based diagnostic and treatment modalities [20]. However, among all participants in each of the three categories, there was a lack of basic biostatistics, which is supported by previous studies that have shown that physicians and pharmacists have limited and poor knowledge of biostatistics and face difficulties in comprehending the value of epidemiological implication [21-24].

While knowledge and skills are a necessity to conduct research, research funding is crucial to cover research expenses and procedures. Our study showed that most participants in "category1 ” lacked grant proposal writing skills. Participants in "category-2" and "category-3" showed poor or very poor grant writing skills. Principally, writing a good research proposal does not mean it will get funded, however, a well-written research proposal can support studies, if approved [25].

The definitive purpose of writing a manuscript is to publish study results and improve knowledge. Indexed journals require a high-quality scientific manuscript for publication. However, writing a manuscript can be exciting and frustrating, which can be discouraging for a novice researcher [26]. The skill level for writing a scientific manuscript between all categories of participants was unsatisfactory, which will lower their chances for publication.

Publishing scientific results is an essential part of a researcher's career. Nevertheless, writing is not every researcher's preferred activity; publishing a scientific article can be boring and timeconsuming [27]. Although the peer review process is unlikely to alter the core nature of a submitted manuscript, in several circumstances, the authors may be required to "add analysis 
or results, clarify thoughts or parameters, revise the statistical testing methods, increase the number of subjects, or lengthen the time of clinical follow-up in response to the reviewer's requests" [28]. Participants in “category-1" showed poor or very poor levels when submitting a manuscript to a journal in terms of fulfilling the journal requirements.

GCP guidelines are recognized internationally as ethical and scientific quality standards. GCP aims to manage and organize the designing, conducting, recording, and reporting of clinical trials involving human subjects. GCP guidelines provide public assurance that the correct safety and well-being of trial subjects are protected and that the data resulting from the trial are complete, accurate, and unbiased [29-30]. Our results revealed a remarkable proportion of poor or very poor knowledge of GCP guidelines among all categories. Although Al-Nomay reported a satisfactory level of GCP knowledge among healthcare professionals in Saudi Arabia, a poor compliance rate was found among study participants [29-30].

Building research capacity is a necessity for public healthcare systems endeavoring to provide outstanding quality care, and evidence-based practice necessitates research to be part of the healthcare system. Education in the above-delineated research skills will shift healthcare providers from untrained or novice researchers to quality researchers and from education to innovation. Our structured set of research skills is common to the success of researchers; it is comprehensive and addresses all research stages. Research skill workshops and education addressing the set of research skills required for success could potentially advance the knowledge of medical professionals regarding research [5].

Research capacity building is the principal operational objective of the KFMC research enterprise. Developing quality researchers and a supportive environment are the main themes of capacity building. Research center services ultimately focusing on providing support in research protocol planning, grant proposal writing, and statistical consultative services for data management create governing and informative policies. In addition, research centers and healthcare environments need to conduct in-service educational workshops related to all aspects of research processes, including infrastructures in the form of research laboratories, equipment, software, and conference support.

The main limitation of our study was that we did not explore the reasons behind poor research knowledge and skills among healthcare providers. Our study aimed to assess research educational needs among healthcare providers from different educational backgrounds. Therefore, it is recommended to conduct future studies exploring potential reasons. Moreover, our study was conducted in a single medical care hospital; hence, conducting a multicenter study is recommended to have a more generalizable result.

\section{Conclusions}

The results obtained from this self-administered questionnaire determine that the educational needs for effective research skills are obvious. The identified educational needs are the cornerstone for constructing a needs-based educational program. Our results showed that there is a significant variation in research skills among researchers; therefore, meeting the educational needs of healthcare providers in order to perform effective research should be constructed based on the appropriate stage of research.

\section{Additional Information}

\section{Disclosures}

Human subjects: Consent was obtained by all participants in this study. King Fahad Medical City issued approval. Animal subjects: All authors have confirmed that this study did not 
involve animal subjects or tissue. Conflicts of interest: In compliance with the ICMJE uniform disclosure form, all authors declare the following: Payment/services info: All authors have declared that no financial support was received from any organization for the submitted work. Financial relationships: All authors have declared that they have no financial relationships at present or within the previous three years with any organizations that might have an interest in the submitted work. Other relationships: All authors have declared that there are no other relationships or activities that could appear to have influenced the submitted work.

\section{References}

1. World Health Organization: World report on knowledge for better health: strengthening health systems. Bull World Health Organ. 2004, 83:

2. Lavis JN, Oxman AD, Moynihan R, Paulsen EJ: Evidence-informed health policy 1 - synthesis of findings from a multi-method study of organizations that support the use of research evidence. Implement Sci. 2008, 3:53. 10.1186/1748-5908-3-53

3. Glencross MJ, Mji AN: The role of a research resource centre in the training of social science researchers. South African J Higher Ed. 2001, 15:179-185. 10.4314/sajhe.v15i2.25370

4. Meerah TS, Osman K, Zakaria E, Ikhsan ZH, Krish P, Lian DK, Mahmod D: Measuring graduate students research skills. Procedia Soc Behav Sci. 2012, 60:626-629.

10.1016/j.sbspro.2012.09.433

5. Khan H, Khawaja MR: Impact of a workshop on the knowledge and attitudes of medical students regarding health research. J Coll Physicians Surg Pak. 2007, 17:59.

6. Aslam F, Shakir M, Qayyum MA: Why medical students are crucial to the future of research in South Asia. PLoS Med. 2005, 2:322. 10.1371/journal.pmed.0020322

7. Pearcey PA: Achieving research-based nursing practice. J Adv Nurs. 1995, 22:33-39. 10.1046/j.1365-2648.1995.22010033.x

8. Pravikoff DS, Tanner AB, Pierce ST: Readiness of US nurses for evidence-based practice: many don't understand or value research and have had little or no training to help them find evidence on which to base their practice. Am J Nurs. 2005, 1:40-51.

9. Koehn ML, Lehman K: Nurses' perceptions of evidence-based nursing practice. J Adv Nurs. 2008, 62:209-215. 10.1111/j.1365-2648.2007.04589.x

10. Potti A, Mariani P, Saeed M, Smego Jr RA: Residents as researchers: expectations, requirements, and productivity. Am J Med. 2003, 115:510-514. 10.1016/j.amjmed.2003.08.017

11. Mylonakis E, Koutkia P: The realities of resident research requirements . JAMA. 1999, 281:2089. 10-1001/pubs.JAMA-ISSN-0098-7484-281-22-jbk0609

12. Leahy N, Sheps J, Tracy CS, Nie JX, Moineddin R, Upshur RE: Family physicians' attitudes toward education in research skills during residency. Can Fam Physician. 2008, 54:413-414.

13. Grant J: Learning needs assessment: assessing the need. BMJ. 2002, 324:156-159. 10.1136/bmj.324.7330.156

14. Kardash CM: Evaluation of undergraduate research experience: perceptions of undergraduate interns and their faculty mentors. J Ed Psych. 2000, 92:191-201. 10.1037/0022-0663.92.1.191

15. Powers DE, Enright MK: Analytical reasoning skills involved in graduate study: perceptions of faculty in six fields. J Higher Ed. 1986, 58:57. 10.1002/j.2330-8516.1986.tb00198.x

16. Bland CJ, Center BA, Finstad DA, Risbey KR, Staples JG: A theoretical, practical, predictive model of faculty and department research productivity. Acad Med. 2005, 80:225-237.

17. Bonaccorsi A, Daraio C: Age effects in scientific productivity . Scientometrics. 2003, 58:49-90.

18. van Arensbergen P, van der Weijden I, Van den Besselaar P: Gender differences in scientific productivity: a persisting phenomenon?. Scientometrics. 2012, 93:857-868. 10.1007/s11192012-0712-y

19. Tower G, Plummer J, Ridgewell B: A multidisciplinary study of gender-based research productivity in the world's best journals. J Diversity Manage. 2007, 2:23-32.

20. Polychronopoulou A, Eliades T, Taoufik K, Papadopoulos MA, Athanasiou AE: Knowledge of European orthodontic postgraduate students on biostatistics. Eur J Orthod. 2010, 33:434-440. 10.1093/ejo/cjq098

21. O'Donnell CA: Attitudes and knowledge of primary care professionals towards evidence-based practice: a postal survey. J Eval Clin Pract. 2004, 10:197-205. 10.1111/j.1365-

2753.2003.00458.x 
22. Estellat C, Faisy C, Colombet I, Chatellier G, Burnand B, Durieux P: French academic physicians had a poor knowledge of terms used in clinical epidemiology. J Clin Epidemiol. 2006, 59:1009-1014. 10.1016/j.jclinepi.2006.03.005

23. Windish DM, Huot SJ, Green ML: Medicine residents' understanding of the biostatistics and results in the medical literature. JAMA. 2007, 298:1010-1022. 10.1001/jama.298.9.1010

24. Bookstaver PB, Miller AD, Felder TM, Tice DL, Norris LB, Sutton SS: Assessing pharmacy residents' knowledge of biostatistics and research study design. Ann Pharmacother. 2012, 46:991-999. 10.1345/aph.10772

25. Tornquist EM, Funk SG: How to write a research grant proposal. J Nurs Scholarsh. 2005, 37:289-293. 10.1111/j.1547-5069.2005.00049.x

26. Singer AJ, Hollander JE: How to write a manuscript . J Emerg Med. 2009, 36:89-93. 10.1016/j.jemermed.2007.09.056

27. Derntl M: Basics of research paper writing and publishing. IJTEL. 2014, 6:105-123. 10.1504/IJTEL.2014.066856

28. Voight ML, Hoogenboom BJ: Publishing your work in a journal: understanding the peer review process. Int J Sports Phys Ther. 2012, 7:452-460.

29. Al-Nomay NS: Compliance with the ICH-GCP guidelines among the Saudi health care professionals: should Saudi Arabia conduct widespread ICH-GCP training?. J Public Health Dev Ctries. 2015, 19:75-82.

30. Al-Nomay NS: Knowledge, perception, and attitude of health care professionals towards ICHGCP guidelines in Saudi Arabia. Avicenna. 2016, 1:1. 10.5339/avi.2016.1 